\title{
Supramolecular arrangement of two methoxy-chalcones
}

Hamilton B. Napolitano, Jean M. F. Custodio, Wesley F. Vaz and Eduardo M Faria Universidade Estadual de Goias

Chalcone molecule is a class of flavonoid intermediates that presents pharmacological importance due their presence in many pharmaceutical compounds ${ }^{1-2}$. The structures of two methoxylated chalcones, namely (E)-1-(4-Meth-oxy-phen-yl)-3-(3,4,5-trimeth-oxy-phen-yl)prop-2-en-1-one, $\mathrm{C}_{19} \mathrm{H}_{20} \mathrm{O}_{5}$ (MT-TRI) and (E)-3-(4-ethoxyphenyl)-1-(4-methoxyphenyl)prop-2-en-1-one, $\mathrm{C}_{18} \mathrm{H}_{18} \mathrm{O}_{3}$ (MT-ETX), reveal the effect of the inclusion of the methoxyl and ethoxyl substituents of the conformation on methoxy-chalcone. Structural comparative study between two chalcones was done in this work and some effects on geometric parameters, such as planarity and dihedral angles, were described. In addition, intermolecular interactions responsible for crystalline packaging were investigated by Hirshfeld surfaces and the values of those interactions were analysed by comparing the experimental and the theoretical models. The theoretical calculations using CAM-B3LYP/6- 311+G(d) systematically over-estimated the IR vibrational spectra and to avoid this we use a scale factor of 0.958 with the objective of better convergence with the experimental results. The spectroscopic data are consistent with the crystal structure. Moreover, the CAM-B3LYP functional showed up to be a good option to obtain the vibrational spectra. The molecular stability was expressed in terms of softness and hardness, both obtained from frontier molecular orbitals. Finally, there is a good agreement between calculated and experimental IR spectrum, which allowed the assignment of the normal vibrational modes.

\section{Acknowledgements}

The authors are grateful to the Brazilian Federal Agency National Counsel of Technological and Scientific Development (CNPq, grant number 313070/2014-8).

\section{References}

1. Bitencourt, H. R.; Guilhon, G. M. S. P.; Arruda, M. S. P.; Marinho, A. M. R.; Santos, A. S.; Brasil, D. S. B.; Santos, L. S. ;J. Braz. Chem. Soc. 2011, 22, 1333.

2. Gaikwad, P.; Priyadarsini, K. I.; Naumov, S.; Rao, B. S. M. ;J. Phys. Chem. A 2010, 114, 7877. 